\title{
Role of Tip60 tumor suppressor in DNA repair pathway
}

\author{
LIU ZhiJian \& SUN YingLi* \\ Beijing Institute of Genomics, Chinese Academy of Sciences, Beijing 100029, China
}

Received December 23, 2010; accepted February 17, 2011

To prevent the damage caused by DNA strand breaks, eukaryotic cells have evolved a series of highly conserved DNA repair mechanisms. The ubiquitously expressed acetyltransferase, Tip60, plays a central role in ATM (ataxia-telangiectasia mutated) activation which is involved in DNA repair. Recent work uncovered a new mechanism of ATM activation mediated by Tip60 and demonstrated that histone methylation, specifically, trimethylation of histone H3, is a key factor in the process. Here, we review the current understanding of how Tip60 is activated and how it activates ATM in response to DNA damage.

Tip60, ATM, MRN, histone methylation, DNA damage, DNA repair

Citation: $\quad$ Liu Z J, Sun Y L. Role of Tip60 tumor suppressor in DNA repair pathway. Chinese Sci Bull, 2011, 56: 1212-1215, doi: 10.1007/s11434-011-4433-Z

Cells are constantly exposed to endogenous (metabolic) and exogenous events (e.g. ionizing and ultraviolet radiation) which can induce potentially harmful DNA damage. To maintain genomic stability and avoid chromosome aberrations, eukaryotic cells have evolved multiple highly conserved DNA repair pathways. This elaborate system integrates DNA damage detection and checkpoint mechanisms to coordinate repair and facilitate cell cycle progression $[1,2]$. The ATM (ataxia-telangiectasia mutated) kinase is a serine/threonine protein kinase and functions as a master regulator for DNA damage response by phosphorylating substrates involved in DNA repair and cell cycle check points [3]. Recent work revealed that Tip60 and mre11-rad50-nbs1 (MRN) complex play a central role in the activation of ATM in response to DNA double-strand breaks (DSBs). Histone H3 trimethylation on lysine 9 is required for activation of the acetyltransferase activity of Tip60 [4]. These results indicate that chromatin modification plays critical role in regulating DNA damage repair.

Tip60 (KAT5) is a ubiquitously expressed acetyltransferase which can acetylate the $\varepsilon$-amino groups of lysine residues on both histone and non-histone proteins, including various targets, such as histone $\mathrm{H} 2 \mathrm{~A}$ and $\mathrm{H} 4$, p53, the ATM

*Corresponding author (email: yinglisun37@gmail.com) kinase etc. [3]. Normal Tip60 expression and activity are critical to multiple signal propagation processes [5]. The finding that homozygous disruption of the Tip60 gene causes early embryonic lethality in mice emphasizes the essential role of Tip60 in embryonic development [6]. Tip60 acts as a tumor suppressor as well. Inactivation of Tip60 can lead to defective DNA repair and increase the risk of cancer [7]. Here, we will focus on advances in our understanding of how Tip60 regulates the DNA repair process.

\section{DNA damage and repair}

DNA damage is harmful and abnormal physical process that can be induced both by environmental factors and normal metabolic processes inside the cell. Accordingly, DNA damage can be divided into 2 major types: endogenous damage and exogenous damage. To maintain the integrity and stability of the genome, mammalian cells have evolved highly complicated mechanisms to detect and repair these types of DNA lesions. Generally, DNA repair processes comprise 3 steps: damage sensing, signaling transduction and repair. However, the efficiency of DNA repair is dependent on many factors, including genetic, epigenetic and 
environmental ones.

Defective DNA repair is strongly correlated with higher risk of cancer in human. Previous work has demonstrated abnormalities in DNA repair processes in different cancers [8]. DNA DSBs are produced by exposure to ionizing radiation, among the most life-threatening types of DNA lesions and particularly difficult to repair. In general, chromosomal DSBs can be repaired by 2 conserved pathways: non-homologous end joining (NHEJ) and homologous recombination (HR) [9].

\section{Tip60 and DNA repair}

Chromatin structure is highly dynamic and critical to almost all DNA-associated processes including gene transcription, replication and repair [10,11]. ATP-dependent remodeling complexes and histone-modifying enzymes are the 2 broad classes of enzymes that are known to regulate chromatin structure. Basically, the first class utilizes energy from ATP hydrolysis to disrupt the contacts between DNA and histones, resulting in alteration of chromatin structure or its components [12]. The second class of enzymes works primarily through covalent modification of histones and induces changes in chromatin structure by altering electrostatic and spacial interactions between DNA and histones [13]. Recent work has revealed that both histone modifications and chromatin remodeling play important role in the cellular response to DNA damage [14]. There is functional crosstalk between modified histones and proteins involved in the DNA damage response (DDR) [4].

Tip60 acetyltransferase is part of the evolutionarily conserved multi-subunit NuA4 complex. Several work has demonstrated that Tip60 was required to maintain genomic integrity and regulate the repair of DNA damage through both transcription-dependent and transcription-independent mechanisms $[15,16]$. The first evidence that Tip60 might have a role in DNA damage response and DNA repair came from transfection experiments in HeLa cells, in which ectopic expression of a mutated Tip60 gene which lacked histone acetylase activity resulted in defective double-strand DNA repair [17]. Similarly, inactivation of Tip60 leads to increased sensitivity to ionizing radiation and an increased level of chromosomal aberrations [4,18]. Furthermore, Tip60 is required for the activation of ATM which is involved in the phosphorylation of several DNA damage response proteins, such as nbs1, p53, chk2 and SMC1 [19,20]. Remarkably, aberrant expression of Tip60 has been detected in various cancers including prostate, breast and colorectal cancer [7,21,22]. These findings showed that Tip60 may impact the efficiency of DNA damage repair, increasing genomic instability and contributing to the initiation and progression of cancers.

\section{Tip60 and ATM activation}

The ATM protein kinase is activated in responses to ionizing radiation in mammalian cells. After DNA damage, ATM is rapidly activated by autophosphorylation on ser1981 which converts it into an active monomer [23]. Activated ATM then catalyzes multiple phosphorylation of downstream substrates which are necessary for proper function of cell-cycle checkpoints and DNA repair [24].

Recent work has demonstrated a direct role of Tip60 in ATM activation [25]. Tip60 HAT (histone acetyltransferase) activity is required for the rapid acetylation and activation of ATM induced by DNA damage. Overexpression of a dominant negative Tip60 mutant in HeLa cells blocks ATM acetylation and prevents the ATM-dependent phosphorylation of H2AX, p53 and ChK2. The same effects were observed with siRNA-mediated knockdown of Tip60. The defective activation of ATM which is caused by lack of Tip60 activity, in turn, greatly sensitizes cells to ionizing radiation. Moreover, the HAT activity of the Tip60-ATM complex is specifically activated by DNA damage. Mutation of ATM acetylation site lys3016 blocks the activation of ATM induced by DNA damage, indicating that Tip60-dependent acetylation of ATM is crucial for the activation of ATM [26]. Sequence comparisons indicate that the ATM acetylation site which lies in the PIKK Regulatory Domain (PRD) is highly conserved among higher eukaryotes [26], whereas the autophosphorylation sites are not $[27,28]$. This difference strongly suggests that acetylation is an evolutionarily conserved event in ATM activation. Interestingly, majority of ATM in the cell forms stable complexes with Tip60 through its conserved FATC domain and the binding is independent of DNA damage [29]. The 33 amino acid FATC domain is required for the activation of ATM. Deletion of the FATC domain blocked the interaction between ATM and Tip60 and therefore suppressed the activation of ATM induced by DNA damage [29].

\section{Tip60 HAT activation}

As discussed before, HAT activity of Tip60 is crucial for ATM activation, a key step for DNA repair. Inactivation of Tip60 leads to defective DNA repair [18] and thus increases the risk of cancer $[22,30]$. Further understanding of how the DNA damage signal activates the acetyltransferase activity of Tip60 and its ability to acetylate ATM is very important. Recent work reported by Price et al. [4] has provided a potential mechanism.

Chromo-domains are specialized binding modules which can interact with methyl groups on methylated lysine residues [31]. There is strong evidence that the chromo-domain of Tip60 binds specifically to histone $\mathrm{H} 3$ trimethylated on 
lysine 9 (H3K9me3). Further study demonstrated that the direct interaction between Tip60 and H3K9me3 at DSBs functions as an allosteric regulator, and activates Tip60 acetyltransferase activity during DNA repair. Disruption of the interaction between Tip60 and H3K9me3 caused either by mutations in the conserved hydrophobic domains of Chromo-domain or inhibition of intracellular H3K9me3 would block activation of the acetyltransferase activity of Tip60 and in turn result in defective ATM activation and in a DNA repair defect. In addition, the ability of Tip60 to access $\mathrm{H} 3 \mathrm{~K} 9 \mathrm{me} 3$ is dependent on the DNA damageinduced release of HP1 $\beta$ (heterochromatin protein $1 \beta$ ) from H3K9me3 [4]. Recent work showed that the Chromo- domain of HP1 $\beta$ is quickly phosphorylated by casein kinase 2 (CK2) after DNA damage occurs. Phosphorylated HP1 $\beta$ then rapidly disassociates from $\mathrm{H} 3 \mathrm{~K} 9 \mathrm{me} 3$ and allows Tip60 to interact with H3K9me3 [32]. Furthermore, the Mre11Rad50-Nbs1 (MRN) complex which targets Tip60 to the proper site in $\mathrm{H} 3 \mathrm{~K} 9 \mathrm{me} 3$ is involved in the initial processing of DNA repair [33,34] and is required for activation of Tip60 acetyltransferase activity. Loss of functional MRN complexes significantly reduces the activation of Tip60's acetyltransferase activity induced by DNA damage [4]. Taken together, these results suggest a three-step model for Tip60 and ATM activation in the DNA damage response
(Figure 1) [3,4].

\section{Conclusion}

Tip60 plays a crucial role in the ATM activation reaction involved in DNA repair processes. Lack of Tip60 activity leads to defective DNA repair and therefore reduces genome stability. The newly discovered mechanism of Tip60 activation suggests that alteration in global histone, especially in H3K9 methylation patterns could affect Tip60 activity and consequently its ability to regulate DNA repair. In view of its functions as a tumor suppressor, the defect of Tip60 activation caused by alteration of histone methylation pattern could theoretically increase the risk of cancer. In fact, aberrant pattern of H3K9 methylation has been reported in both gastric adenocarcinoma [35] and medulloblastoma [36]. In addition, genetic alteration of histone methyltransferases, including Dot1, Suv4-20h and Suv49 $\mathrm{h} 1 / \mathrm{h} 2$ [37-39], results in aberrant histone methylation, genomic instability, checkpoint activation and DNA repair [3,38-40]. In summary, we propose that alteration of histone methylation pattern may contribute to the initiation and progression of cancer by influencing the activation of the ATM-Tip60 pathway in response to DNA damage. Based

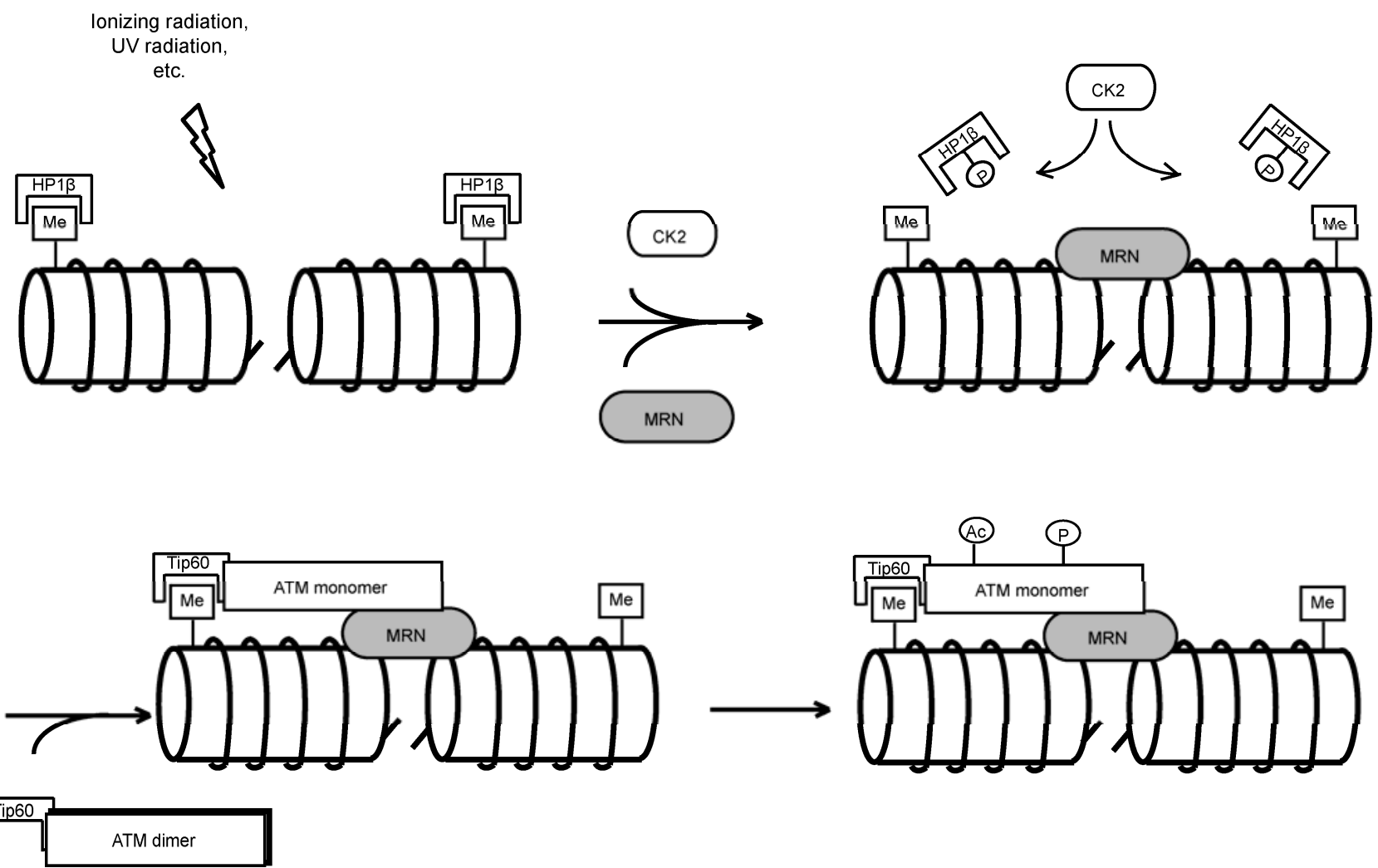

Figure 1 Three-step model for Tip60 and ATM activation. Step1: Following occurrence of DSBs, MRN complex and CK2 are recruited to the DSB site. Meanwhile, CK2 phosphorylates HP1 $\beta$ leading to the release of HP1 $\beta$ from H3K9me3. Step2: Tip60-ATM complex is recruited to MRN. The Chromo-domain of Tip60 interacts with exposed H3K9me3. Step3: Tip60 acetyltransferase activity is activated and in turn results in the activation of ATM. Me, methylation; Ac, acetylation; p, phosphorylation [3,4]. 
on the observation that alteration in the distribution of $\mathrm{H} 3 \mathrm{~K} 9 \mathrm{me} 3$ across the chromatin during tumor progression may significantly impact genomic stability and affect the sensitivity of tumors to chemo- and radio-therapy, the strategy of epigenetic targeting at $\mathrm{H} 3 \mathrm{~K} 9 \mathrm{me} 3$ could be an effective tool for cancer treatment and potentially protect normal tissues from DNA damage.

This work was supported by the Hundred Talents Program of the Chinese Academy of Sciences and the National Natural Science Foundation of China (91019024).

1 Hoeijmakers $\mathbf{J}$ H. Genome maintenance mechanisms for preventing cancer. Nature, 2001, 411: 366-374

2 Lobrich M, Jeggo P A. The impact of a negligent G2/M checkpoint on genomic instability and cancer induction. Nat Rev Cancer, 2007, 7: 861-869

3 Sun Y L, Jiang X, Price B D. Tip60: Connecting chromatin to DNA damage signaling. Cell Cycle, 2010, 9: 930-936

4 Sun Y L, Jiang X F, Xu Y, et al. Histone H3 methylation links DNA damage detection to activation of the tumour suppressor Tip60. Nat Cell Biol, 2009, 11: 1376-1382

5 Carrozza M J, Utley R T, Workman J L, et al. The diverse functions of histone acetyltransferase complexes. Trends Genet, 2003, 19: 321-329

$6 \mathrm{Hu}$ Y, Fisher J B, Koprowski S, et al. Homozygous disruption of the Tip60 gene causes early embryonic lethality. Dev Dyn, 2009, 238: 2912-2921

7 Gorrini C, Squatrito M, Luise C, et al. Tip60 is a haplo-insufficient tumour suppressor required for an oncogene-induced DNA damage response. Nature, 2007, 448: 1063-1067

8 Kosti O, Goldman L, Saha D T, et al. DNA damage phenotype and prostate cancer risk. Mutat Res, 2010, 719: 46-46

9 Pardo B, Gomez-Gonzalez B, Aguilera A. DNA repair in mammalian cells: DNA double-strand break repair: How to fix a broken relationship. Cell Mol Life Sci, 2009, 66: 1039-1056

10 Peterson C L, Cote J. Cellular machineries for chromosomal DNA repair. Genes Dev, 2004, 18: 602-616

11 Shivaswamy S, Iyer V R. Stress-dependent dynamics of global chromatin remodeling in yeast: Dual role for SWI/SNF in the heat shock stress response. Mol Cell Biol, 2008, 28: 2221-2234

12 Lusser A, Kadonaga J T. Chromatin remodeling by ATP-dependent molecular machines. Bioessays, 2003, 25: 1192-1200

13 Kouzarides T. Chromatin modifications and their function. Cell, 2007, 128: 693-705

14 van Attikum H, Gasser S M. The histone code at DNA breaks: A guide to repair? Nat Rev Mol Cell Biol, 2005, 6: 757-765

15 Squatrito M, Gorrini C, Amati B. Tip60 in DNA damage response and growth control: Many tricks in one HAT. Trends Cell Biol, 2006, 16: 433-442

16 Niida H, Katsuno Y, Sengoku M, et al. Essential role of Tip60-dependent recruitment of ribonucleotide reductase at DNA damage sites in DNA repair during $\mathrm{G}_{1}$ phase. Genes Dev, 2010, 24: 333-338

17 Ikura T, Ogryzko V V, Grigoriev M, et al. Involvement of the Tip60 histone acetylase complex in DNA repair and apoptosis. Cell, 2000, 102: 463-473

18 Murr R, Loizou J I, Yang Y G, et al. Histone acetylation by Trrap-Tip60 modulates loading of repair proteins and repair of DNA double-strand breaks. Nat Cell Biol, 2006, 8: 91-99

19 Sancar A, Lindsey-Boltz L A, Unsal-Kacmaz K, et al. Molecular mechanisms of mammalian DNA repair and the DNA damage checkpoints. Annu Rev Biochem, 2004, 73: 39-85

20 Lavin M F, Birrell G, Chen P, et al. ATM signaling and genomic stability in response to DNA damage. Mutat Res, 2005, 569: 123-132

21 Halkidou K, Gnanapragasam V J, Mehta P B, et al. Expression of Tip60, an androgen receptor coactivator, and its role in prostate cancer development. Oncogene, 2003, 22: 2466-2477

22 Sakuraba K, Yasuda T, Sakata M, et al. Down-regulation of Tip60 gene as a potential marker for the malignancy of colorectal cancer. Anticancer Res, 2009, 29: 3953-3955

23 Bakkenist C J, Kastan M B. DNA damage activates ATM through intermolecular autophosphorylation and dimer dissociation. Nature, 2003, 421: 499-506

24 Bakkenist C J, Kastan M B. Initiating cellular stress responses. Cell, 2004, 118: 9-17

25 Sun Y L, Jiang X, Chen S, et al. A role for the Tip60 histone acetyltransferase in the acetylation and activation of ATM. Proc Natl Acad Sci USA, 2005, 102: 13182-13187

26 Sun Y L, Xu Y, Roy K, et al. DNA damage-induced acetylation of lysine 3016 of ATM activates ATM kinase activity. Mol Cell Biol, 2007, 27: 8502-8509

27 Daniel J A, Pellegrini M, Lee J H, et al. Multiple autophosphorylation sites are dispensable for murine ATM activation in vivo. J Cell Biol, 2008, 183: 777-783

28 Pellegrini M, Celeste A, Difilippantonio S, et al. Autophosphorylation at serine 1987 is dispensable for murine Atm activation in vivo. Nature, 2006, 443: 222-225

29 Jiang X, Sun Y L, Chen S, et al. The FATC domains of PIKK proteins are functionally equivalent and participate in the Tip60-dependent activation of DNA-PKcs and ATM. J Biol Chem, 2006, 281: 15741-15746

30 Shiota M, Yokomizo A, Masubuchi D, et al. Tip60 promotes prostate cancer cell proliferation by translocation of androgen receptor into the nucleus. Prostate, 2010, 70: 540-554

31 Taverna S D, Li H, Ruthenburg A J, et al. How chromatin-binding modules interpret histone modifications: Lessons from professional pocket pickers. Nat Struct Mol Biol, 2007, 14: 1025-1040

32 Ayoub N, Jeyasekharan A D, Bernal J A, et al. HP1-beta mobilization promotes chromatin changes that initiate the DNA damage response. Nature, 2008, 453: 682-686

33 Uziel T, Lerenthal Y, Moyal L, et al. Requirement of the MRN complex for ATM activation by DNA damage. EMBO J, 2003, 22: 5612-5621

34 Chailleux C, Tyteca S, Papin C, et al. Physical interaction between the histone acetyl transferase Tip60 and the DNA double-strand breaks sensor MRN complex. Biochem J, 2010, 426: 365-371

35 Park Y S, Jin M Y, Kim Y J, et al. The global histone modification pattern correlates with cancer recurrence and overall survival in gastric adenocarcinoma. Ann Surg Oncol, 2008, 15: 1968-1976

36 Northcott P A, Nakahara Y, Wu X, et al. Multiple recurrent genetic events converge on control of histone lysine methylation in medulloblastoma. Nat Genet, 2009, 41: 465-472

37 Peters A H, O'Carroll D, Scherthan H, et al. Loss of the Suv39h histone methyltransferases impairs mammalian heterochromatin and genome stability. Cell, 2001, 107: 323-337

38 Wysocki R, Javaheri A, Allard S, et al. Role of Dot1-dependent histone $\mathrm{H} 3$ methylation in G1 and S phase DNA damage checkpoint functions of Rad9. Mol Cell Biol, 2005, 25: 8430-8443

39 Sakaguchi A, Steward R. Aberrant monomethylation of histone H4 lysine 20 activates the DNA damage checkpoint in Drosophila melanogaster. J Cell Biol, 2007, 176: 155-162

40 Giannattasio M, Lazzaro F, Plevani P, et al. The DNA damage checkpoint response requires histone H2B ubiquitination by Rad6Bre1 and H3 methylation by Dot1. J Biol Chem, 2005, 280: 98799886

Open Access This article is distributed under the terms of the Creative Commons Attribution License which permits any use, distribution, and reproduction in any medium, provided the original author(s) and source are credited. 